\title{
Editorial
}

La revista Cuadernos de Lingüística Hispánica, órgano de difusión de la Maestría en Lingǘstica de la Universidad Pedagógica y Tecnológica de Colombia (Uptc), recientemente reindexada en Categoría B por Publindex-Colciencias, llega hoy a su número 26 correspondiente al período julio-diciembre de 2015, otra meta alcanzada gracias a la colaboración permanente de nuestro equipo editorial. En esta entrega divulgamos la productividad académica de estudiantes y docentes investigadores nacionales e internacionales. Los artículos que aquí aparecen se enmarcan en las tres líneas de investigación que desarrolla el plan de estudios de este Programa de formación posgraduada, a saber: Lenguaje y sociedad, Pedagogía del lenguaje, y Lenguaje y comunicación; con el apoyo en su Proyecto Académico Educativo (PAE), presentamos una somera descripción de cada una:

\section{Lenguaje y sociedad}

La fundamentación teórica de esta línea fue elaborada por el profesor Donald Freddy Calderón Noguera, exdirector de la Maestría en Linguiística de la Uptc, quien señala que se enmarca en los procesos investigativos que aluden al estudio de la relación existente entre el lenguaje y los procesos sociales, desde las interdisciplinas: Sociolingüística, Sociología del lenguaje, Etnolingüística, Etnografía de la comunicación, Lenguas en contacto y otras similares. Se busca comprender las interacciones fundamentales, complementarias y auxiliares del papel que cumple el lenguaje como comportamiento desde la perspectiva funcional. El giro lingüístico orientó el papel de los estudios del lenguaje apoyados en una perspectiva inmanentista, estructural y disciplinar hacia una concepción del lenguaje en uso capaz de trascender las diferentes dimensiones de lo humano. Hoy el lenguaje y su teoría lingüística atraviesan o inciden considerablemente en los estudios científicos 
de varias disciplinas, hasta el punto de hablarse de la lingüística como método para las ciencias sociales.

El objetivo de esta línea es consolidar el estado del arte del lenguaje y su relación con la sociedad, en los contextos local, regional, nacional e internacional mediante proyectos de investigación que aborden problemas teóricos, empíricos y metodológicos tendientes a explorar, describir, explicar y teorizar desde el lenguaje asuntos relacionados con inclusión, participación y pertenencia en conglomerados sociales. Entre los niveles en los que se apoyan las diversas investigaciones destacan: socio fonético-fonológico, sociosintáctico, socio-léxico, semántico, socio-discursivo y socio-pragmático. Así, tienen espacio los temas relacionados con usos dialectales; variación y cambio; lenguas en contacto, pidgins, criollos; identidad, inclusión, participación y pertenencia social; pertinencia, eficacia, interferencia y fracaso comunicativo, entre otros.

Las investigaciones pueden realizarse de manera exclusiva, dominante y mixta en los siguientes marcos, modelos y enfoques:

Marcos: histórico hermenéutico y lógico positivo.

Modelos: diacrónico, sincrónico, cualitativo y cuantitativo.

Enfoques: variacionista y etnográfico.

\section{Pedagogía del lenguaje}

Su base teórica fue construida por José Humberto Motta Ávila, docente de la Maestría en Lingüística; él expone allí lo siguiente: esta línea se constituye en una estrategia de producción, difusión y aplicación de conocimientos necesarios para la solución de problemas reales relacionados con la enseñanza y el aprendizaje de la lengua española, en los niveles de Preescolar, Educación Básica, Educación Media y Educación Superior. Dado que la Maestría en Lingüística, de la Facultad de Ciencias de la Educación, de la Uptc, hace énfasis especial en la Lengua castellana, lo que a continuación se expone ha de entenderse como referido a dicho marco contextual. La línea Pedagogía del lenguaje se plantea en consonancia con la postura filosófica y epistemológica que afirma que más que una filosofía del lenguaje y una epistemología de la lingüística, de lo que se trata es de abordar una filosofía y una epistemología de las ciencias del lenguaje. Esto, dados los rasgos de complejidad e interdisciplinariedad del estudio del lenguaje y las lenguas. De otra parte, la consideración de la Pedagogía como un campo científico (Bedoya, 2005) e interdisciplinario y como el saber fundante de la praxis pedagógica, complementa los asideros macro sobre los que se fundamenta la línea mencionada. La línea subsume los conceptos disciplinares propios de la Lingüística y la Pedagogía, -como interdisciplinas- 
desde los más recientes hallazgos gnoseológicos. Así, por ejemplo, la comprensión de lenguaje, lingüística, lengua, cultura, comunicación, pedagogía, didáctica, currículo, enseñanza, aprendizaje, conocimiento, es abordada desde la óptica de un conocimiento multidimensional, como lo propone Morín (2004).

Igualmente, la comprensión del acto didáctico se hace desde la pluralidad de propuestas contemporáneas que lo muestran como un hecho complejo realizable a través de una forma variopinta. Las didácticas contemporáneas, nacidas unas en el seno de la pedagogía y otras en espacios disciplinares diferentes, iluminan la riqueza de procedimientos con la que cuenta el docente para desplegar su creatividad e innovación. En este caso, se hace referencia a didáctica problémica, aprendizaje basado en problemas, estructuración cognitiva, aprendizaje significativo, enseñanza para la comprensión, inteligencias múltiples, pedagogía conceptual, pedagogía afectiva (Zubiría, 2006); trabajo por proyectos, pedagogía experiencial, entre otras. De tal manera, la línea contribuye con la integración curricular de la Maestría, posibilitando la interdisciplinariedad y la transdisciplinariedad, lo cual reduce la fragmentación del currículo para estimular los niveles más complejos de pensamiento y ayudar a los magistrandos a integrar el conocimiento.

De este modo, la fundamentación teórica y epistemológica de la línea se convierte en un campo abierto y flexible, de permanente actualización -al ritmo de los avances-, propicio para satisfacer los procesos de producción de conocimiento y de intervención pedagógica. Como se indicó en el apartado anterior, la línea está abierta a la diversidad teórica y metodológica. Esto quiere decir que tanto los orientadores de los seminarios, como los participantes, pueden seleccionar, del surtido de posibilidades, aquellas alternativas disciplinares, interdisciplinares o transdisciplinares que son acordes con la naturaleza del conocimiento o del procedimiento que estén tratando. Esto, debido a que las distintas ciencias del lenguaje han creado sus propios marcos teóricos que satisfacen las necesidades de conocimiento para la resolución de sus problemas pertinentes.

Al igual que en el componente teórico conceptual, la forma de hacer investigación en la pedagogía de las ciencias del lenguaje se abre en abanico, por la similar razón de que cada disciplina ha creado sus propios métodos; y en el encuentro con la pedagogía, tales métodos se actualizan y combinan con los de las alternativas pedagógico-didácticas, generando así una síntesis creativa de múltiples determinaciones. De esta forma, una investigación pedagógica en procesos de lectura dará resultados disímiles si se trata con la propuesta de la Enseñanza para la Comprensión o con la propuesta de la Pedagogía Afectiva. 
Son objetivos de esta línea: contribuir a la formación de una masa crítica que haga nuevas lecturas del currículo oficial colombiano, en la perspectiva de proponer diseños curriculares alternativos para el área de lenguaje; aplicar criterios teórico-prácticos para la elaboración de diseños curriculares de aula, como modelos de aprendizaje-enseñanza en el área de lenguaje; promover y desarrollar la investigación en áreas de la Linguística Aplicada a fin de contribuir a la solución de problemas actuales de interés local, regional y nacional; fomentar la generación y la aplicación de conocimientos en Linguiística Aplicada para el mejoramiento de las prácticas pedagógicas relacionadas con el lenguaje, en los niveles institucional, local, regional y nacional; crear productos informáticos que incorporen algún componente en el que intervenga el lenguaje oral o escrito; desarrollar la competencia investigativa, en los magistrandos, para formular proyectos que propendan hacia el mejoramiento de las competencias de lectura, oralidad, escucha y escritura en los contextos educativos.

\section{Lenguaje y comunicación}

El sustento teórico de esta tercera línea de investigación fue preparado por Gloria Smith Avendaño de Barón, exdirectora de la Maestría en Lingüística de la Uptc. En su disertación advierte que el objeto de estudio de esta Maestría es la linguística del lenguaje que de acuerdo con Lucía Tobón (2007) es un estudio del proceso de significar, o la capacidad para hallar relación entre una estructura ausente (idea, pensamiento, sentimiento) y una representación que la hace presente en la conciencia de los interlocutores; sin olvidar que no solo mediante la palabra se puede significar, pues existe una gran diversidad de señales, signos y símbolos que también pueden cumplir esta función; es decir, se significa mediante el lenguaje verbal y el no verbal. Y todo ser humano, en condiciones normales, es capaz de producir sistemas sígnicos que le permiten transmitir su pensamiento a la mente de otros. Entonces, la función específica de la facultad de lenguaje es concretar el acto de significar que va de la abstracción a la representación.

Lucía Tobón insiste en que el objeto de estudio de la Lingüística es el lenguaje: «Es entre las facultades de la mente humana, la que tiene que ver con el proceso que va de cualquier actividad perceptiva de hechos o fenómenos sensoriales, emotivos e, incluso, kinésicos, y de concepciones intelectuales abstractas a la conceptualización y representación» (p. 60). Así, la lingüística deja de ser una ciencia dirigida solo al estudio de la lengua, propósito que le concierne a la gramática, para adoptar también el estudio de otros sistemas simbólicos de representación y de comunicación. A la linguística también le compete señalar las condiciones que deben cumplir los distintos sistemas simbólicos para servir de vehículos de los procesos de comunicación en su doble vía: producción y recepción de mensajes; fijar pautas para el estudio y análisis de los diferentes sistemas 
semióticos, estén ellos constituidos por notaciones, señales, signos o símbolos; definir los estratos o dimensiones semántica, sintáctica y pragmática que presentan en su estructura, y si en verdad se puede hablar de códigos sin semántica o sin semiótica, pues por tratarse de representaciones mentales deben registrar un substrato y una impronta cultural que les sirvan de soporte en su tarea de reproducir lo que la mente crea como resultado de procesos cognitivos, de vivencias afectivas o de experiencias alcanzadas en contextos individuales o sociales.

En síntesis, esta línea de investigación sincretiza la competencia comunicativa con la competencia lingüística y se interesa en el análisis del lenguaje que se emplea en los medios masivos de comunicación, desde diversas representaciones y perspectivas.

Para efectos de investigación en el campo del lenguaje y la comunicación, se centra la atención en los paradigmas Interpretativo y Crítico, y se adoptan los métodos teórico, experimental e instrumental. En ellos se trata de hacer empíricamente accesibles los mecanismos de la comunicación general -social y massmediática- y lingüística -verbal y paraverbal- (Jorques, 2004). La línea Lenguaje y comunicación acoge los enfoques de investigación cuantitativos y cualitativos, de manera combinada, para la construcción de modelos o teorías referidas al papel que desempeña el lenguaje en los diversos medios de comunicación.

Los siguientes son los propósitos centrales de esta línea: propiciar la discusión sobre el papel del lenguaje en los procesos comunicativos mediados por la diversidad de signos, códigos y símbolos que el ser humano crea para aprehender y categorizar la realidad en la búsqueda del acceso, comprensión y producción del conocimiento; incentivar procesos de aproximación crítico-científica al funcionamiento del lenguaje articulado y del lenguaje de la imagen, como sistemas de significación en situaciones comunicativas; promover el análisis del lenguaje empleado por los medios de comunicación; estimular procesos de teorización sobre la estructura, recepción y producción del lenguaje utilizado en la creación de blogs, páginas web, libretos de radio y de televisión, entre otros medios; motivar la capacidad de crear modelos teóricos orientados a la dinamización del pensamiento crítico a partir del lenguaje de los medios de comunicación; orientar procesos que posibiliten determinar y caracterizar los diversos discursos que circulan en las Tecnologías de la Información y la Comunicación; y promover la reflexión permanente sobre el carácter multimodal del lenguaje.

En este contexto linguístico, la edición número 26 de nuestra revista presenta los siguientes artículos: "Los nombres propios hispánicos en Camerún: entre aventura y globalización semiolingüística"; "Traducción y cultura: reflexiones sobre la dimensión 
cultural de textos y su importancia para la traducción"; "Didáctica y Lingüística: un desafío desde la universidad para la educación básica"; "Traer estativo en español de México: su estructura sintáctica y propiedades semánticas"; "Su sabor provoca... análisis de transitividad y subjetividad en anuncios publicitarios"; "Tres indigenismos en el parlache: concho, cucha/cucho y tote"; "Ideología y reconocimiento de los pueblos indígenas: análisis del discurso político del Consejo Regional Indígena del Cauca (CRIC)"; "Prácticas discursivas orales, dialectos e identidad social"; "Estrés escolar y empatía en estudiantes de bachillerato practicantes de Mindfulness" y "Discurso pedagógico en maestros desde lo enunciado por estudiantes de colegios católicos".

Los trabajos precitados corresponden a productos derivados de proyectos de investigación inscritos en grupos, tales como: "Esquemas Sintáctico-Semánticos de traer y llevar en uso estativo-posesivo", de la Universidad Autónoma de Querétaro, UAQ, México; "Estudios del lenguaje y la comunicación" (Categoría A, Colciencias), de la Universidad Tecnológica de Pereira, Colombia; "Textos creativos y la identidad social en Colombia", de la Universidad Distrital "Francisco José de Caldas"; "Grupo Origen”, de la Universidad del Rosario, Colombia.

Nos complace resaltar que en este número incluimos contribuciones enviadas por investigadores de universidades extranjeras; por ejemplo: Universidad de Maroua, Camerún; Universidad de Ciencias Aplicadas de Colonia, Alemania; Universidad Autónoma de Querétaro, México; Universidad Pedagógica Experimental Libertador-El Mácaro, sede Aragua (Venezuela) y Universidad de Buenos Aires (UBA). Igualmente, destacamos las contribuciones de docentes y estudiantes de instituciones de educación superior públicas y privadas de Colombia, entre otras: Universidad Tecnológica de Pereira; Universidad del Norte, Barranquilla; Universidad Industrial de Santander (UIS); Universidad de Antioquia; Universidad del Rosario; Universidad Distrital "Francisco José de Caldas"; Fundación universitaria "Los Libertadores", Bogotá y Universidad Pedagógica y Tecnológica de Colombia.

No es posible culminar sin expresar un sincero agradecimiento a cada uno de los autores de los distintos artículos; a los comités editorial, científico y de árbitros; a la doctora Olga Nájar Sánchez, decana de la Facultad de Ciencias de la Educación (Uptc); al doctor Hugo Alfonso Rojas Sarmiento, director de la DIN; a la profesora Bertha Ramos Holguín, Editora en jefe -Unidad Editorial; al doctor Francisco Leguizamón, director del Centro de Investigación y Extensión de la Facultad de Ciencias de la Educación (CIEFED); al coordinador académico de la Maestría en Linguística, profesor Joselyn Corredor Tapias; a las docentes traductoras, Imperio Arenas González (lengua francesa), Juliana Borrero (lengua inglesa) y Neila Wolff (lengua portuguesa); al maestrando Alfredo de Jesús 
Mendoza Escalante, asistente editorial; a la señora Ana Joaquina Ríos Nope, secretaria de la Maestría en Lingüística y a cada uno de los lectores de nuestras ediciones semestrales.

Invitamos a los investigadores en el campo del lenguaje a remitir sus contribuciones en uno de los siguientes idiomas: español, inglés, francés y portugués. Es oportuno informar que la convocatoria para su envío está permanentemente abierta.

Gloria Smith Avendaño de Barón

Editora 